\title{
INFLUÊNCIA DAS CARACTERÍSTICAS DAS SUBSTÂNCIAS HÚMICAS AQUÁTICAS NA EFICIÊNCIA DA COAGULAÇÃO COM O CLORETO FÉRRICO
}

\author{
Eliane Sloboda* e Eny Maria Vieira \\ Instituto de Química de São Carlos, Universidade de São Paulo, Av. Trabalhador São-Carlense, 400, 13566-570 São Carlos - SP, \\ Brasil \\ Angela Di Bernardo Dantas e Luiz Di Bernardo \\ Departamento de Hidráulica e Saneamento, Escola de Engenharia de São Carlos, Universidade de São Paulo, Av. Trabalhador \\ São-Carlense, 400, 13566-570 São Carlos - SP, Brasil
}

Recebido em 22/7/08; aceito em 13/11/08; publicado na web em 26/2/09

\begin{abstract}
THE INFLUENCE OF AQUATIC HUMIC SUBSTANCES CHARACTERISTICS ON THE COAGULATION EFFICIENCY USING FERRIC CHLORIDE. The aim of this study was to verify the influence of the apparent molecular size of aquatic humic substances on the effectiveness of coagulation with ferric chloride. Coagulation-filtration tests using jar test and bench-scale sand filters were carried out on samples of water with true color of approximately 100 Hazen units, prepared with aquatic humic substances of different molecular sizes $\left(\mathrm{F}_{1}:<0.45 \mu \mathrm{m}, \mathrm{F}_{2}: 100 \mathrm{kDa}-0.45 \mu \mathrm{m}, \mathrm{F}_{3}: 30-100 \mathrm{kDa}\right.$ and $\left.\mathrm{F}_{4}:<30 \mathrm{kDa}\right)$. For the water samples with lower apparent molecular size fractions, greater dosages of coagulant was needed to remove the color around 5.0 Hanzen units, mainly because these water samples contain higher concentrations of fulvic acids, which exhibited a larger number of negatively-charged groups.
\end{abstract}

Keywords: aquatic humic substances; apparent molecular size; color removal.

\section{INTRODUÇÃO}

A matéria orgânica natural (MON) presente em águas naturais pode ser subdividida em matéria orgânica dissolvida (MOD) e matéria orgânica particulada (MOP). A MOD é operacionalmente definida como a fração que atravessa o filtro com poros de $0,45 \mu \mathrm{m}$, e essa fração constitui a maior parte da matéria orgânica presente em águas naturais (aproximadamente 80\%). A MOD presente em águas naturais possui vários compostos orgânicos, como proteínas, aminoácidos, ácidos graxos, polímeros e substâncias húmicas ( $\mathrm{SH}$ ), sendo que a maior parte está na forma $\mathrm{SH}$, às quais correspondem cerca de 50 a $70 \%$ da MOD. ${ }^{1}$

Thurman ${ }^{2}$ concluiu em seus estudos que a MOP é a matéria orgânica proveniente de zooplancton e algas, retida na membrana de $0,45 \mu \mathrm{m}$. A MOP corresponde a aproximadamente $17 \%$ da MON presente em águas naturais e é usualmente removida pelos métodos convencionais de tratamento de água, como coagulação, filtração e ajuste do $\mathrm{pH} .^{3}$

As SH são encontradas em solos, solos turfosos, sedimentos e águas naturais; são formadas pela degradação química e biológica de resíduos de vegetais e de animais e da atividade de síntese de microrganismos..$^{2-4}$

As SH podem ser classificadas como substâncias orgânicas biogênicas, polieletrolíticas com propriedades similares às de biocolóides, de coloração escura, de natureza heterogênea, possuem elevada massa molecular e estrutura complexa. ${ }^{5}$ Schulten e Schnitzer ${ }^{6}$ e Swift ${ }^{7}$ propuseram que as SH são macromoléculas e Diallo et al. ${ }^{8}$ propuseram que as SH possuem estrutura supramolecular. Porém, sua estrutura ainda é indefinida.

As substâncias húmicas aquáticas (SHA) podem ser formadas diretamente no meio aquático por decomposição de plantas e pela atividade sintética de microrganismos e/ou as $\mathrm{SH}$ encontradas em solos e sedimentos podem ser transportadas para as águas naturais por

*e-mail: elisloboda@hotmail.com processos de escoamento superficial. ${ }^{9}$ Thurman e Malcolm ${ }^{10}$ definiram as SHA como sendo a porção não específica, amorfa, constituída de MOD em pH 2 e adsorvente em coluna de resina XAD 8, não-iônica, com altos valores de coeficientes de distribuição.

A classificação das SHA de acordo com a solubilidade em diferentes condições de $\mathrm{pH}$ divide-se em frações de ácidos fúvicos (AF), ácidos húmicos $(\mathrm{AH})$ e humina. Os AF são solúveis em toda faixa de $\mathrm{pH}$; os $\mathrm{AH}$ são solúveis em valores de $\mathrm{pH} \geq 2$ e a humina é a fração insolúvel em toda faixa de $\mathrm{pH} .{ }^{11}$ As três frações húmicas diferem quanto à massa molecular e quantidade de grupos funcionais, tendo os AF massa molecular mais baixa, maior quantidade porcentual de oxigênio e menor conteúdo de carbono e nitrogênio que as outras frações húmicas. ${ }^{12}$

Ao contrário das substâncias húmicas extraídas de turfa, que apresentam predominância de AH, as SHA contêm maior quantidade de AF. No geral, as SHA possuem elevado tamanho molecular aparente, podendo variar desde $200 \mathrm{Da}$ até cerca de $100 \mathrm{kDa}$, estando os AF entre 200 e $2000 \mathrm{Da}$, enquanto que dos AH é maior que $2000 \mathrm{Da} .{ }^{12}$ Thurman et al. ${ }^{13}$ sugerem que esta variação de tamanho molecular aparente seja devida à fonte das $\mathrm{SH}$, à metodologia analítica usada e à agregação das $\mathrm{SH}$.

A presença das SH é a principal responsável pela cor escura nas águas naturais. Devido a essas características, a maior parte das estações de tratamento de água (ETA) apresenta problemas de operação, pelo fato da tecnologia de tratamento empregada não se adequar à qualidade da água a ser tratada, requerendo o uso da pré-oxidação, principalmente para a redução de cor. ${ }^{12}$

Quando as SHA não são eficientemente removidas durante as etapas de tratamento de água, podem causar alguns problemas como, servir de substrato para o crescimento de microrganismos; reduzir a eficiência do processo de desinfecção; complexar metais, como Mn, $\mathrm{Pb}$ e Fe e outros, dificultando suas remoções; produzir substâncias com sabor e odor desagradáveis, algumas tóxicas e carcinogênicas quando a pré-oxidação das SH é feita com cloro livre. ${ }^{12}$

A MON de águas superficiais pode ser removida por diferentes 
processos, tais como, coagulação, utilização de membranas (microfiltração, ultrafiltração, nanofiltração), osmose reversa, oxidação, biofiltração, resinas de troca iônica e carvão ativado. ${ }^{14}$

A coagulação é considerada como sendo uma etapa de grande importância no tratamento da água, a qual é feita por meio da adição de produtos químicos apropriados, seguida de mistura rápida. Usualmente são utilizados sais de alumínio ou de ferro ou polímeros sintéticos como coagulantes. A função desses coagulantes é desestabilizar partículas pequenas, geralmente de tamanho coloidal carregadas negativamente, remover a MOD, induzir a floculação e melhorar a filtração. ${ }^{15}$

Durante o processo de coagulação usando sais de ferro, as SH carregadas negativamente podem interagir com as espécies hidrolisadas positivas do metal, formando complexos insolúveis na forma de humatos ou fulvatos (mecanismo neutralização de cargas) ou podem ser adsorvidas no precipitado de hidróxido de ferro (mecanismo de varredura). ${ }^{16}$

O mecanismo de varredura é mais apropriado quando a coagulação é feita antes da floculação e remoção de flocos por sedimentação ou flotação. O mecanismo de adsorção e neutralização de carga é mais efetivo quando as ETA empregam uma das tecnologias de filtração direta, pois não há necessidade de formação de flocos para posterior sedimentação ou flotação, mas sim de partículas desestabilizadas que são retidas no meio granular dos filtros. ${ }^{12}$

Dantas et al. ${ }^{17}$ avaliaram o efeito do tamanho molecular aparente das SHA na coagulação com sulfato de alumínio de quatro águas de estudo preparadas com cor verdadeira igual a $100 \mathrm{uH}$. Os autores concluíram que águas com a mesma coloração, mas com SHA com propriedades estruturais distintas, podem resultar em diferentes condições de coagulação.

A presença de grupos funcionais, aromaticidade, variação no tamanho molecular, proporção de AH e AF nas SHA influenciam na eficiência da remoção das $\mathrm{SH}$ presentes na água. ${ }^{18} \mathrm{O}$ uso de diferentes técnicas analíticas (ultrafiltração, espectroscopia de RMN, no UV/ VIS e no infravermelho, por exemplo) é de grande importância no entendimento das características estruturais das SH. Este conhecimento ajuda a compreender os resultados obtidos durante o tratamento da água por coagulação.

O presente trabalho foi desenvolvido com o objetivo de comparar os resultados obtidos na coagulação com cloreto férrico e filtração direta, para águas preparadas com SHA de diferentes tamanhos moleculares aparentes e diferentes características estruturais das SHA.

\section{PARTE EXPERIMENTAL}

\section{Coleta e extração das substâncias húmicas aquáticas}

As SHA utilizadas neste estudo para caracterização e preparação das águas de estudo foram extraídas de aproximadamente 2.000 L de água coletada no Rio Itapanhaú, Bertioga-SP, Brasil. Foram analisados os principais parâmetros físicos e químicos da amostra de água coletada.

Para a extração das SHA foi utilizada a resina macroporosa não iônica de éster acrílico (XAD 8). Foi utilizado o procedimento que a International Humic Substances Society (IHSS) tem adotado para a extração de SHA. ${ }^{19}$

A água bruta foi acidificada com $\mathrm{HCl}(\mathrm{pH} \cong 2,5)$ e percolada através de coluna de acrílico preenchida com $30 \mathrm{~cm}$ de resina XAD 8 , com vazão de aproximadamente $22 \mathrm{~mL} \mathrm{~min}^{-1}$. A saturação da resina foi verificada pela medida da absorbância em $254 \mathrm{~nm}$.

A eluição das SHA foi feita empregando-se solução de $\mathrm{NaOH}$ $0,10 \mathrm{~mol} \mathrm{~L}^{-1}$, com vazão aproximadamente de 5,0 $\mathrm{mL} \mathrm{min}{ }^{-1}$. Após a eluição, fez-se o tratamento da resina com metanol e lavagens sucessivas com água para ser reutilizada.
O extrato de SHA final foi submetido em solução de $\mathrm{HCl}$ a pH 2,0 até atingir um valor de $\mathrm{pH}$ em torno de 6,0. A seguir, foi feita a diálise dos extratos com água não clorada para remoção do excesso de sais dissolvidos, até teste negativo de cloretos (teste com solução de nitrato de prata $\left.0,10 \mathrm{~mol} \mathrm{~L}^{-1}\right)$. $\mathrm{O}$ pH final do extrato ficou em torno de 5,0-6,0.

\section{Fracionamento das SHA por ultrafiltração (UF) e liofilização}

O extrato de SHA primeiramente foi diluído com água deionizada na proporção de 1:4 e, em seguida, filtrado a vácuo em membrana de $0,45 \mu \mathrm{m}$ (Millipore) para posterior fracionamento por UF (membranas de polyethersulfane Vivaflow 50 com fluxo tangencial, Vivascience). Durante o fracionamento das SHA, foi adotado o método de concentração da amostra por recirculação. ${ }^{20} \mathrm{~A}$ máxima vazão utilizada foi de $300 \mathrm{~mL} \mathrm{~min}^{-1}$ com pressão de 1,5 bar.

Foram obtidos os extratos com diferentes tamanhos moleculares aparentes: $\mathrm{F}_{1}$ : menor que $0,45 \mu \mathrm{m}, \mathrm{F}_{2}$ : entre $100 \mathrm{kDa}$ e $0,45 \mu \mathrm{m}, \mathrm{F}_{3}$ : entre 30 e $100 \mathrm{kDa}, \mathrm{F}_{4}$ : entre 10 e $30 \mathrm{kDa} ; \mathrm{F}_{5}$ : entre 5 e $10 \mathrm{kDa}$ e $\mathrm{F}_{6}$ : menor que $5 \mathrm{kDa}$. A $\mathrm{F}_{6}$ não foi caracterizada devido à necessidade de um volume grande para se conseguir a massa necessária.

Parte do extrato das SHA foi concentrado em evaporador rotativo e liofilizado em liofilizador Heto. As frações liofilizadas foram caracterizadas por análises espectroscópicas no infravermelho, ultravioleta/visível, ressonância magnética nuclear e análise elementar. Outra parte foi utilizada para preparar as águas de estudo usadas nos ensaios de coagulação.

\section{Análise elementar}

Foi determinada a composição elementar das SHA em relação ao conteúdo de carbono $(\mathrm{C})$, hidrogênio $(\mathrm{H})$, nitrogênio $(\mathrm{N})$ e enxofre (S) em equipamento para análise elementar CHNSO-CE Instruments, Perkin Elmer, EA 1110. O oxigênio foi obtido por diferença.

\section{Teor de cinzas}

Amostras de aproximadamente $10 \mathrm{mg}$ dos extratos liofilizados foram calcinadas a $800{ }^{\circ} \mathrm{C}$ em mufla marca EDG, modelo 1800-3P, durante $4 \mathrm{~h}$. Foi calculado o teor de matéria orgânica pela diferença de massa após a calcinação, considerando-se o resíduo final a matéria inorgânica.

\section{Espectroscopia no infravermelho com transformada de Fourier}

As análises de infravermelho foram feitas em um espectrômetro com transformada de Fourier, marca Bomem MB-102. Para a análise foram feitas pastilhas do material liofilizado na proporção de 1-100 (1 $\mathrm{mg}$ de amostra e $100 \mathrm{mg}$ de $\mathrm{KBr}$ ). Foram feitas 16 varreduras com $4 \mathrm{~cm}^{-1}$ de resolução nos espectros obtidos na região de 400 a $4.000 \mathrm{~cm}^{-1}$.

\section{Espectroscopia na região do ultravioleta (UV)}

As análises de UV foram feitas em um espectrômetro de UV/ VIS Hitachi modelo U3501. Para a análise dissolveu-se $2 \mathrm{mg}$ de SHA em 25 mL de solução de bicarbonato de sódio $\left(\mathrm{NaHCO}_{3}\right)$ 0,05 mol L ${ }^{-1}$. Determinou-se as razões $\mathrm{E}_{2} / \mathrm{E}_{3}$, nos comprimentos de onda de 250 e $365 \mathrm{~nm}$.

\section{Ressonância magnética nuclear (RMN)}

A análise de RMN foi feita em um espectrômetro de RMN da marca Varian Unit Inova, operando a uma freqüência de 100 e 400 
$\mathrm{MHz}$ para ${ }^{13} \mathrm{C}$. Foi utilizada uma rampa de radiofreqüência na polarização cruzada, velocidade de rotação da amostra em torno do ângulo mágico de $13 \mathrm{kHz}$ e desacoplamento em alta potência (CP/MAS).

\section{Porcentagem de AH e AF nas frações de SHA}

Os extratos fracionados em diferentes tamanhos moleculares aparente de SHA foram acidificados com $\mathrm{HCl} \mathrm{em} \mathrm{pH}$ menor que 2,0 e decantação por $24 \mathrm{~h}$. Após a precipitação do AH, as amostras foram centrifugadas em $4000 \mathrm{rpm}$ por $30 \mathrm{~min}$ e, em seguida, foi coletado o sobrenadante para medidas de carbono orgânico total (COT). As medidas do COT foram feitas em um equipamento de carbono orgânico total TOC-V CPH/CPN/P da Shimadzu, pelo método de combustão seguido de leitura no infravermelho.

\section{Preparação das águas de estudo}

Para a preparação das águas de estudo foi utilizada água do poço artesiano localizado no campus da USP/São Carlos e extratos de SHA fracionados em diferentes tamanhos moleculares aparentes. Foram preparados aproximadamente $250 \mathrm{~L}$ de cada água de estudo, todas com cor verdadeira de $100 \pm 5 \mathrm{uH}$, turbidez em torno de $5 \mathrm{uT}$ (com adição de caulinita) e pH em torno de 6,0 (ajustado com uma solução de ácido clorídrico $0,1 \mathrm{M}$ ). A água de estudo 1 foi preparada com a $\mathrm{F}_{1}$ (SHA passada na $0,45 \mu \mathrm{m}$ ), a água de estudo 2 foi preparada com a $F_{2}(100 \mathrm{kDa}-0,45 \mu \mathrm{m})$, a água de estudo 3 foi preparada com a $\mathrm{F}_{3}$ (30-100 kDa) e a água de estudo 4 foi preparada com a $\mathrm{F}_{4}$ ' (menor que $30 \mathrm{kDa}$ ). Não foram estudadas separadamente as frações, $\mathrm{F}_{4}$ (entre 10 e $30 \mathrm{kDa}$ ), $\mathrm{F}_{5}$ (entre 5 e $10 \mathrm{kDa}$ ) e $\mathrm{F}_{6}$ (menor que $5 \mathrm{kDa}$ ) para preparação das águas de estudo, devido à necessidade de um grande volume dessas frações para obtenção de cor verdadeira da água de $100 \mathrm{uH}$. Sendo assim, a $\mathrm{F}_{4}{ }^{\prime}$ contém todas essas frações.

\section{Ensaios de coagulação e filtração direta}

Para as quatro águas de estudo foram construídos os diagramas de coagulação, variando-se a dosagem do coagulante cloreto férrico líquido como produto comercial (contém $38 \%$ de $\mathrm{FeCl}_{3} \quad 6 \mathrm{H}_{2} \mathrm{O}$ ) e o pH de coagulação. Para alterar o pH de coagulação foram utilizadas soluções de hidróxido de sódio $(\mathrm{NaOH}) 4 \mathrm{~g} \mathrm{~L}^{-1}$ e de ácido clorídrico ( $\mathrm{HCl} 1 \mathrm{~g} \mathrm{~L}^{-1}$. A temperatura da água de estudo foi mantida constante em todos os ensaios, em 20,0 $\pm 1,0^{\circ} \mathrm{C}$.

Todos os ensaios foram feitos em equipamento de jarteste, nas seguintes condições: mistura rápida (tempo de agitação $=30 \mathrm{~s}$; gradiente de velocidade médio $=1000 \mathrm{~s}^{-1}$ ) e filtração em filtros de areia em escala laboratorial (areia com tamanho dos grãos entre 0,30 e $0,59 \mathrm{~mm}$ ), tempo de filtração de 20 min e taxa média de filtração de 50 a $70 \mathrm{~m}^{3} / \mathrm{m}^{2}$ dia.

Os parâmetros de controle durante os ensaios de coagulação foram: cor aparente da água filtrada (espectrofotômetro DR-4000Hach), turbidez da água filtrada (turbidímetro $2100 \mathrm{~N}$ da Hach) e $\mathrm{pH}$ de coagulação (potenciômetro da Orion).

\section{RESULTADOS E DISCUSSÃO}

\section{Caracterização da água bruta}

Foram determinados alguns parâmetros da água coletada no Rio Itapanhaú. Os resultados foram: $\mathrm{pH}=5,6$; cor aparente $=300 \mathrm{uH}$; cor verdadeira $=279 \mathrm{uH}$; absorbância $(254 \mathrm{~nm})=1,6$; turbidez $=2,78 \mathrm{uT}$ e alcalinidade total $=6,0 \mathrm{mg} \mathrm{L}^{-1} \mathrm{CaCO}_{3}$. A água bruta foi acidificada com ácido clorídrico até $\mathrm{pH} \cong 2,5$ para evitar a decomposição da matéria orgânica durante o tempo de armazenamento e, também, para permitir a protonação dos grupos funcionais das SHA e conseqüente adsorção na resina macroporosa XAD 8.

\section{Análise elementar}

A Tabela 1 apresenta a composição elementar em porcentagem e as razões atômicas nas frações das SHA.

Tabela 1. Análise elementar das SHA e razões atômicas

\begin{tabular}{lccccccc}
\hline \multirow{2}{*}{ Frações } & \multicolumn{3}{c}{ \% Massa dos elementos } & \multicolumn{3}{c}{ Razões atômicas } \\
& $\mathrm{C}$ & $\mathrm{H}$ & $\mathrm{N}$ & $\mathrm{O} *$ & $\mathrm{H} / \mathrm{C}$ & $\mathrm{O} / \mathrm{C}$ & $\mathrm{C} / \mathrm{N}$ \\
\hline $\mathrm{F}_{1}(<0,45 \mu \mathrm{m})$ & 37,35 & 3,34 & 1,44 & 57,86 & 1,07 & 1,16 & 30,23 \\
$\mathrm{~F}_{2}(<0,45 \mu \mathrm{m}$ a & 36,01 & 3,32 & 1,29 & 59,38 & 1,10 & 1,24 & 32,57 \\
$100 \mathrm{kDa})$ & & & & & & & \\
$\mathrm{F}_{3}(100$ a $30 \mathrm{kDa})$ & 37,44 & 3,56 & 0,91 & 58,10 & 1,14 & 1,16 & 48,10 \\
$\mathrm{~F}_{4}(30 \mathrm{a} 10 \mathrm{kDa})$ & 37,95 & 4,34 & 0,80 & 56,90 & 1,37 & 1,12 & 55,20 \\
$\mathrm{~F}_{5}(10$ a $5 \mathrm{kDa})$ & 35,53 & 4,51 & 0,70 & 59,30 & 1,52 & 1,25 & 58,96 \\
\hline
\end{tabular}

* A porcentagem de oxigênio foi calculada por diferença.

Observa-se pelos dados apresentados na Tabela 1, que a fração $\mathrm{F}_{5}$ apresentou as menores porcentagens de $\mathrm{C}$ e $\mathrm{N}$ e as porcentagens de $\mathrm{H}$ e $\mathrm{O}$ relativamente aumentaram com a diminuição do tamanho molecular aparente. Isto pode indicar que as frações de menor tamanho molecular aparente contêm maior conteúdo de carbonos alifáticos do que aromáticos e uma quantidade relativamente alta de oxigênios ligados a grupamentos alquílicos e de ácidos carboxílicos. ${ }^{21}$ Não foi encontrado enxofre nas frações das SHA estudadas. Verificou-se que a razão $\mathrm{H} / \mathrm{C}$ aumentou gradativamente com a diminuição do tamanho molecular aparente, o que pode indicar que as menores frações possuem um menor grau de anéis aromáticos.

\section{Teor de cinzas}

As porcentagens de cinzas nas frações das SHA com diferentes tamanhos moleculares aparentes foram: $\mathrm{F}_{1}=29,68 ; \mathrm{F}_{2}=25,24 ; \mathrm{F}_{3}=$ 15,$30 ; \mathrm{F}_{4}=15,92$ e $_{5}=17,43$.

O teor de cinzas é geralmente um indicativo do conteúdo de compostos inorgânicos e/ou grau de pureza. Porém, a presença de complexos de cálcio, magnésio e sílica pode elevar os teores de cinzas. Também a presença de compostos de fósforo, nem sempre volatilizados durante a queima, pode estar incluída nas cinzas. ${ }^{22}$

Outro fator que pode elevar os teores de cinzas é que a maioria dos valores expressos na literatura é para SH fracionada em $\mathrm{AH}$ e AF e purificada com resinas de troca aniônica (IR-400) e catiônica (IR-120). ${ }^{23,24}$ Este procedimento não foi realizado neste estudo com o objetivo de obter o material húmico na forma mais natural possível, evitando possíveis alterações das principais características das SHA.

\section{Análise espectroscópica no infravermelho com transformada de Fourier}

Na Figura 1 são apresentados os espectros na região do infravermelho das frações de SHA de diferentes tamanhos moleculares aparentes.

Os espectros de infravermelho (Figura 1) apresentam bandas largas, comuns nos espectros de $\mathrm{SH}$, atribuídas às superposições de absorções individuais e similaridades entre os grupos funcionais presentes nas estruturas das $\mathrm{SH}$. 


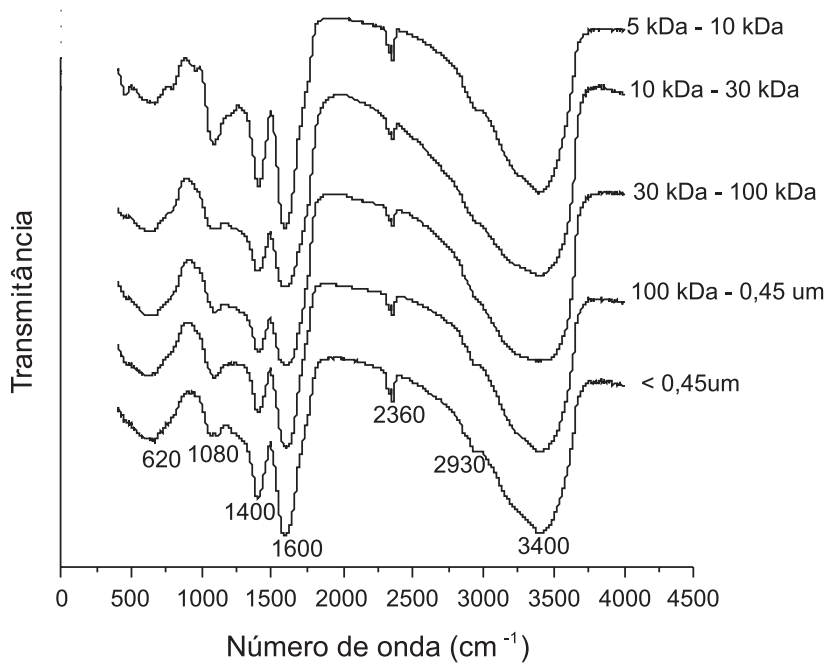

Figura 1. Análise espectroscópica no infravermelho das frações de SHA

Observam-se bandas de absorção largas e intensas para todas as frações das SHA, muito peculiar em torno de $3400 \mathrm{~cm}^{-1}$, que são atribuídas ao estiramento $\mathrm{OH}$ de grupamentos carboxílicos, fenólicos, alcoólicos e da água ligada. As bandas em aproximadamente 2900 $\mathrm{cm}^{-1}$ são atribuídas ao estiramento $\mathrm{C}-\mathrm{H}$ dos grupos metil ou metilenos alifáticos, geralmente superpostos à banda larga de estiramento $\mathrm{OH}$. As bandas em $1600 \mathrm{~cm}^{-1}$ indicam aromaticidade das SHA, característica do estiramento $\mathrm{C}=\mathrm{C}$ do anel, isto é, às vibrações do anel aromático conjugado com $\mathrm{C}=\mathrm{O}$ e/ou $\mathrm{COO}^{-}$. Essas bandas podem também ser devidas ao estiramento anti-simétrico do grupo carboxilato e ao estiramento $\mathrm{C}=\mathrm{O}$ do grupo $\mathrm{COOH}$, sendo que neste caso o hidrogênio está ligado ao grupo $\mathrm{OH}$ em posição orto. Entre 1370 e $1440 \mathrm{~cm}^{-1}$ observam-se bandas devidas a grupos $\mathrm{C}-\mathrm{H}$ alifáticos e vibração $\mathrm{OH}$ de álcoois ou ácidos carboxílicos. ${ }^{11-25}$ Isso pode confirmar a menor aromaticidade das SHA, já que a maior porcentagem é de AF.

Comparando-se os espectros das frações de diferentes tamanhos moleculares, verifica-se que são praticamente os mesmos, indicando grandes similaridades entre os grupos funcionais presentes nas estruturas das SHA.

\section{Análise espectroscópica na região do ultravioleta (UV)}

A Tabela 2 lista os resultados da razão $\mathrm{E}_{2} / \mathrm{E}_{3}$ de frações de diferentes tamanhos moleculares aparentes de SHA.

Tabela 2. Características espectroscópicas de UV/VIS para as frações das SHA

\begin{tabular}{lcccc}
\hline Frações & \multicolumn{2}{c}{ Absorbância $(\mathrm{nm})$} & Razão & $\begin{array}{c}\text { Aroma- } \\
\text { ticidade }\end{array}$ \\
\hline $\mathrm{F}_{1}(<0,45 \mu \mathrm{m})$ & 250 & 365 & $\mathrm{E}_{2} / \mathrm{E}_{3}$ & \\
$\mathrm{~F}_{2}(<0,45 \mu \mathrm{m}$ a $100 \mathrm{kDa})$ & 0,449 & 0,146 & 3,06 & 31,75 \\
$\mathrm{~F}_{3}(100$ a $30 \mathrm{kDa})$ & 1,124 & 0,361 & 3,11 & 31,41 \\
$\mathrm{~F}_{4}(30$ a $10 \mathrm{kDa})$ & 0,680 & 0,198 & 3,43 & 29,24 \\
$\mathrm{~F}_{5}(10 \mathrm{a} 5 \mathrm{kDa})$ & 0,658 & 0,166 & 3,94 & 25,78 \\
\hline
\end{tabular}

De acordo com a Tabela 2 observa-se que os valores das razões $\mathrm{E}_{2} / \mathrm{E}_{3}$ aumentaram à medida que diminuiu o tamanho molecular aparente, enquanto que para a aromaticidade, ocorreu o inverso. Isso mostra que as frações de maior tamanho molecular aparente possuem maior quantidade de anéis aromáticos condensados em comparação às estruturas alifáticas, em decorrência de um alto grau de humificação do material húmico.

Utilizou-se a equação sugerida por Peuravuori e Pihlaja ${ }^{26}$ para indicar a aromaticidade da molécula, onde: aromaticidade $=52,5-$ $6,78 \cdot \mathrm{E}_{2} / \mathrm{E}_{3}$.

\section{Ressonância magnética nuclear de ${ }^{13} \mathrm{C}$ no estado sólido (RMN de $\left.{ }^{13} \mathrm{C}\right)$}

A Figura 2 apresenta os espectros de RMN CP/MAS de ${ }^{13} \mathrm{C}$ das frações de diferentes tamanhos moleculares aparentes das SHA.
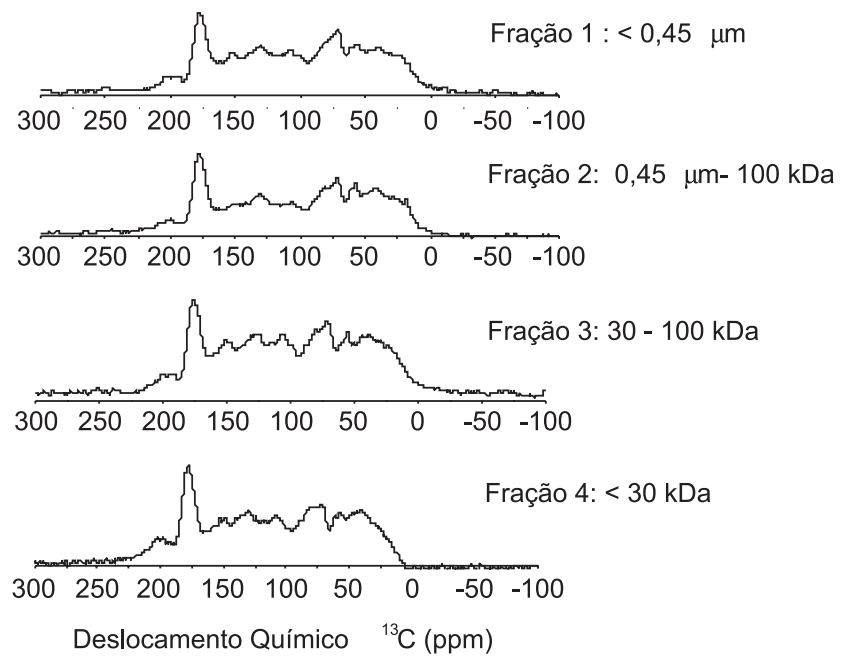

Figura 2. Espectros de $R M N$ de ${ }^{13} C$ para as frações das $S H A$

Analisando-se os espectros de RMN apresentados na Figura 2, verifica-se que apresentam os picos bastante similares, porém não se pode comparar a intensidade dos picos devido à utilização de tamanhos moleculares aparentes diferentes.

Na região de 0 a 50 ppm foram observados picos similares para todas as frações, o que representa a ressonância de carbonos alifáticos (metil, metileno). ${ }^{27} \mathrm{~A}$ presença de grupos alifáticos na fração de maior tamanho molecular aparente pode ser em decorrência do tamanho dessas moléculas, com agregados que possuem grande concentração de cadeias aromáticas ligadas a carbonos lineares. Esses dados estão de acordo com a proposta da estrutura dos $\mathrm{AH}$ obtida por meio dos espectros de RMN feita por Schulten e Schiltzer. ${ }^{6}$ Segundo os autores, os AH são formados por grupos aromáticos com longas cadeias alifáticas ligadas.

Na região de 50 a 90 ppm observam-se picos similares para todas as frações, o que representa a porcentagem relativa de carbonos alifáticos oxigenados. ${ }^{27}$ Como observado na Figura 3, a fração de SHA com tamanho molecular aparente menor que 0,45 $\mu$ m (fração geral das SHA) apresentou maior porcentagem de AF que AH. Conforme a proposta feita por Piccolo, ${ }^{28}$ os AF são formados por uma maior quantidade de grupos com oxigênio ligados, o que lhes proporcionaria um caráter repulsivo com a formação de agregados menores do que dos AH. Na região de 162 a 190 ppm está relacionada aos grupos carboxílicos, amida e ésteres. ${ }^{29-31}$ Os picos presentes na região de 71 a 73 ppm são atribuídos às cadeias de polissacarídeos, como a celulose. ${ }^{32}$ Porém, como pode ser verificado na Figura 2, não foi possível observar esses picos. Na região dos aromáticos (90-162 ppm) observa-se que as porcentagens relativas foram proporcionais para todas as frações.

A região de 140-160 ppm é referente à ressonância de carbonos aromáticos substituídos por oxigênio e nitrogênio, como fenóis, éteres 
aromáticos ou aminas. ${ }^{31}$ Verifica-se pela Figura 2 que apenas as frações $\mathrm{F}_{1}, \mathrm{~F}_{3}$ e $\mathrm{F}_{4}$ apresentaram um pico mais intenso em torno de 150 ppm. Os picos presentes na região de 190 a 250 ppm são atribuídos a carbonos de aldeídos e cetonas. ${ }^{31}$ Nesta região foram observados picos menos intensos para ambas as frações das SHA.

Na Tabela 3 são apresentadas as porcentagens (\%) das intensidades relativas de diferentes regiões espectrais (ppm) de RMN, para as frações de SHA com diferentes tamanhos moleculares aparentes. As porcentagens foram obtidas mediante a integração de intervalos das regiões espectrais (deslocamento químico de ${ }^{13} \mathrm{C}$, ppm) e normalizadas pela área total do espectro.

Tabela 3. Porcentagem $(\%)$ das intensidades relativas em relação às diferentes regiões espectrais (ppm) de RMN para as frações de SHA com diferentes tamanhos moleculares aparentes

\begin{tabular}{|c|c|c|c|c|c|}
\hline \multirow{3}{*}{ Frações de SHA } & \multicolumn{5}{|c|}{$\begin{array}{l}\text { Porcentagem }(\%) \text { das intensidades relativas em } \\
\text { relação às diferentes regiões espectrais de RMN }\end{array}$} \\
\hline & \multicolumn{5}{|c|}{ Deslocamento químico de ${ }^{13} \mathrm{C}, \mathrm{ppm}$} \\
\hline & $0-50$ & $50-90$ & $90-162$ & $162-190$ & $190-250$ \\
\hline$<0,45 \mu \mathrm{m}$ & 19,0 & 22,7 & 32,6 & 15,1 & 6,1 \\
\hline $100 \mathrm{kDa}-0,45 \mu \mathrm{m}$ & 20,2 & 23,7 & 30,0 & 16,3 & 6,7 \\
\hline $30-100 \mathrm{kDa}$ & 18,7 & 20,0 & 31,9 & 13,2 & 6,5 \\
\hline$<30 \mathrm{kDa}$ & 16,9 & 21,8 & 34,2 & 16,8 & 8,7 \\
\hline
\end{tabular}

É possível observar a partir da Tabela 3 que a intensidade relativa na região espectral de 0-50 ppm (grupos alquil e metoxil) diminuiu para as frações de menores tamanhos moleculares aparentes.

$\mathrm{Na}$ região de 50-90 ppm (O-alquil) as porcentagens foram similares para todas as frações. O comportamento contrário (aumento da intensidade em função do tamanho molecular aparente) é observado nas regiões de 90-162 e 162-190 ppm. Isso mostra um aumento da quantidade de grupos di-O-alquil e aromáticos substituídos por oxigênios e nitrogênios, grupos carboxílicos e ésteres, respectivamente.

\section{Porcentagem de carbono orgânico total}

Como observado na Figura 3, na fração entre $100 \mathrm{kDa}$ e 0,45 $\mu$ m prevaleceram os $\mathrm{AH}(92,4 \%)$, e nas frações abaixo de $30 \mathrm{kDa}$ prevaleceram os $\mathrm{AF}(100 \%)$.

Portanto, como as SHA foram extraídas utilizando a resina XAD 8, o material húmico obtido é constituído na maior parte por frações hidrofóbicas.

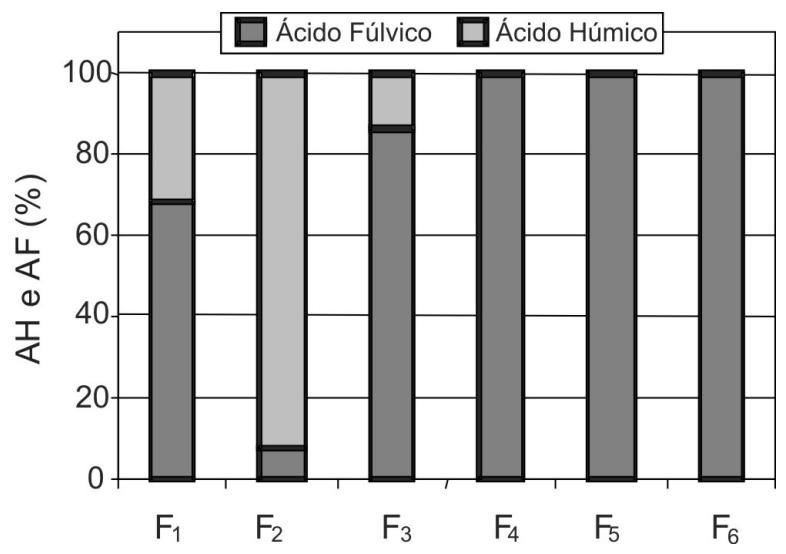

Figura 3. Porcentagem de AH e AF nas diferentes frações das SHA

\section{Caracterização da água do poço artesiano e das águas de estudo}

A caracterização da água do poço artesiano utilizada no preparo das águas de estudo pode ser verificada na Tabela 4.

Tabela 4. Algumas características físicas e químicas da água do poço artesiano

\begin{tabular}{lc}
\hline Características & Valores \\
\hline Turbidez $(\mathrm{uT})$ & $<0,2$ \\
Cor aparente $(\mathrm{uH})$ & $<2,0$ \\
$\mathrm{pH}$ & $6,4 \mathrm{a} 6,6$ \\
Ferro $\left(\mathrm{mg} \mathrm{L}^{-1}\right)$ & $<0,05$ \\
Manganês $\left(\mathrm{mg} \mathrm{L}^{-1}\right)$ & $<0,01$ \\
Cromo $\left(\mathrm{mg} \mathrm{L}^{-1}\right)$ & $\mathrm{ND}$ \\
Cádmio $\left(\mathrm{mg} \mathrm{L}^{-1}\right)$ & $\mathrm{ND}$ \\
Cobalto $\left(\mathrm{mg} \mathrm{L}^{-1}\right)$ & $\mathrm{ND}$ \\
Níquel $\left(\mathrm{mg} \mathrm{L}^{-1}\right)$ & $\mathrm{ND}$ \\
Alumínio $\left(\mathrm{mg} \mathrm{L}^{-1}\right)$ & $\mathrm{ND}$ \\
Zinco $\left(\mathrm{mg} \mathrm{L}^{-1}\right)$ & $\mathrm{ND}$ \\
\hline
\end{tabular}

ND: não detectado

Como pode ser observado na Tabela 4, a água do poço é praticamente isenta de cor, turbidez e metais. Essas são as principais características que justificam o seu uso no preparo das águas de estudo e, também, a ausência de interferentes no processo de coagulação das substâncias húmicas.

Na Tabela 5 são apresentados alguns parâmetros físicos e químicos determinados nas águas de estudo de importância neste estudo.

Pode-se observar na Tabela 5, com exceção do carbono orgânico dissolvido, que as características físicas e químicas resultaram similares para as 4 águas de estudo. O COD da água de estudo 4 (fração de $\mathrm{SHA}<30 \mathrm{kDa}$ ) foi da ordem de 2 vezes maior que nas águas de estudo 2 e 3 (frações de maior tamanho molecular aparente). Isso,

Tabela 5. Algumas características das amostras de água de estudo, para a realização dos ensaios de coagulação com o cloreto férrico

Valores

\begin{tabular}{|c|c|c|c|c|}
\hline Características & $\begin{array}{l}\text { Água de } \\
\text { estudo } 1\end{array}$ & $\begin{array}{l}\text { Água de } \\
\text { estudo } 2\end{array}$ & $\begin{array}{l}\text { Água de } \\
\text { estudo } 3\end{array}$ & $\begin{array}{l}\text { Água de } \\
\text { estudo } 4\end{array}$ \\
\hline $\begin{array}{l}\text { Tamanho molecular } \\
\text { aparente }\end{array}$ & $\mathrm{F}_{1}$ & $\mathrm{~F}_{2}$ & $\mathrm{~F}_{3}$ & $\mathrm{~F}_{4}^{\prime}$ \\
\hline $\begin{array}{l}\text { Condutividade } \\
\text { elétrica }(\mu \mathrm{s} / \mathrm{cm})\end{array}$ & 79,10 & 83,00 & 81,90 & 87,70 \\
\hline Cor verdadeira $(\mathrm{uH})$ & $100 \pm 5$ & $100 \pm 5$ & $100 \pm 5$ & $100 \pm 5$ \\
\hline Cor aparente $(\mathrm{uH})$ & 137 a 151 & 130 a 136 & 151 a 156 & 130 a 146 \\
\hline $\mathrm{pH}$ & $6,0 \pm 0,1$ & $6,0 \pm 0,1$ & $6,0 \pm 0,1$ & $6,0 \pm 0,1$ \\
\hline Temperatura $\left({ }^{\circ} \mathrm{C}\right)$ & $20,0 \pm 1,0$ & $20,0 \pm 1,0$ & $20,0 \pm 1,0$ & $20,0 \pm 1,0$ \\
\hline Turbidez (uT) & $5,0 \pm 0,5$ & $5,0 \pm 0,5$ & $5,0 \pm 0,5$ & $5,0 \pm 0,5$ \\
\hline $\begin{array}{l}\text { Alcalinidade total } \\
\left(\mathrm{mg} \mathrm{L}^{-1}\right)\end{array}$ & 10,00 & 10,60 & 9,00 & 11,00 \\
\hline $\mathrm{COD}^{*}\left(\mathrm{mg} \mathrm{L}^{-1}\right)$ & 10,55 & 5,30 & 7,59 & 16,00 \\
\hline
\end{tabular}


provavelmente, ocorreu devido à adição de um volume elevado de extrato de SHA com tamanho molecular aparente menor que $30 \mathrm{kDa}$, para preparar a água de estudo 4 com cor de $100 \mathrm{uH}$, conseqüentemente, a massa de carbono nessa fração foi maior.

\section{Diagramas de coagulação com cloreto férrico}

Nas Figuras 4 a 7 são apresentados os resultados dos ensaios de coagulação e filtração direta para as diferentes dosagens de cloreto

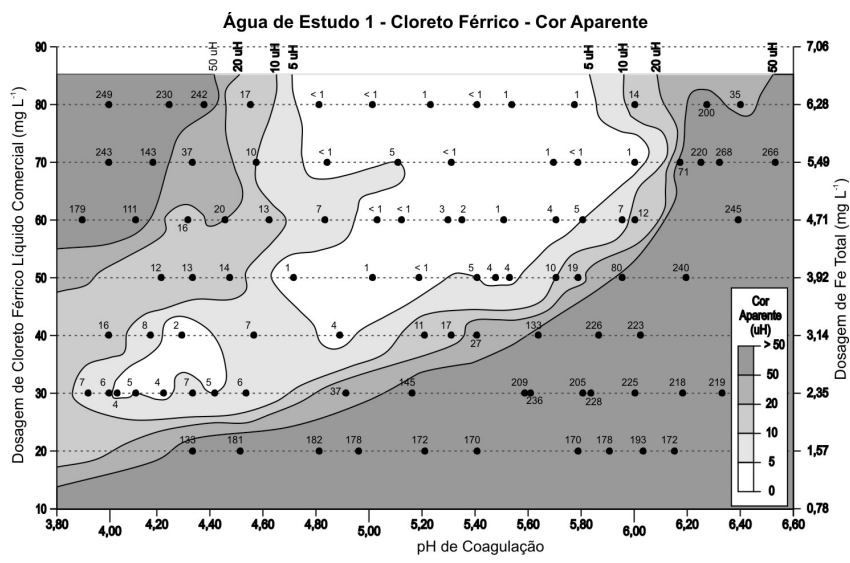

Figura 4. Diagrama de coagulação com cloreto férrico para água de estudo 1 (fração de SHA menor que 0,45 $\mu \mathrm{m}$ )

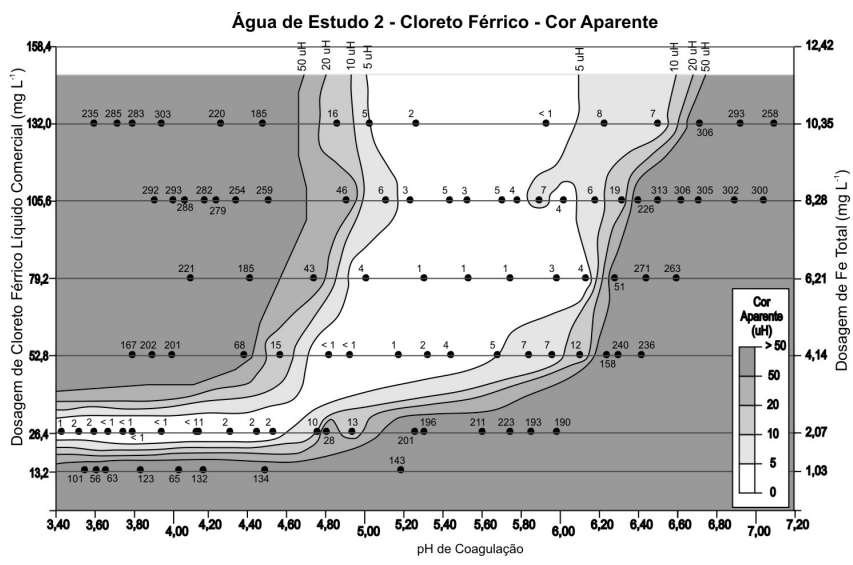

Figura 5. Diagrama de coagulação com cloreto férrico para água de estudo 2 (fração de SHA entre $100 \mathrm{kDa}$ e 0,45 $\mu \mathrm{m}$ )

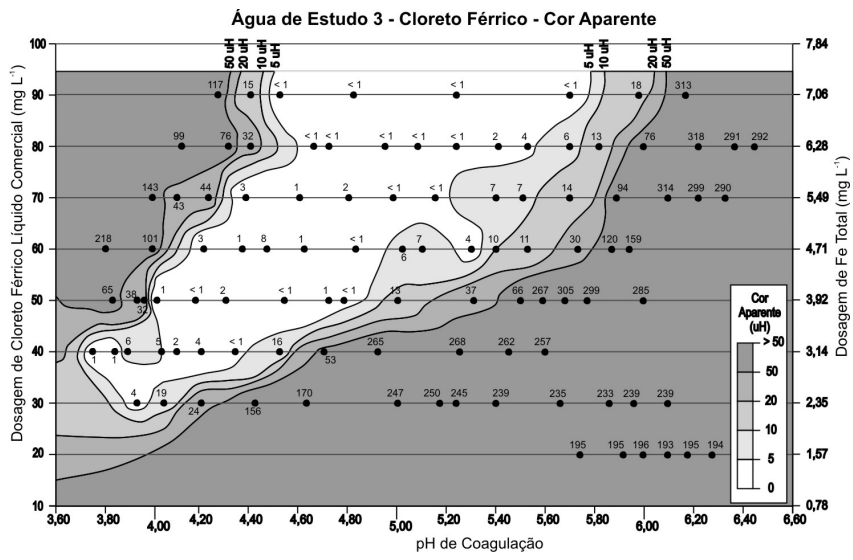

Figura 6. Diagrama de coagulação com cloreto férrico para água de estudo 3 (fração de SHA entre 30 e $100 \mathrm{kDa}$ )

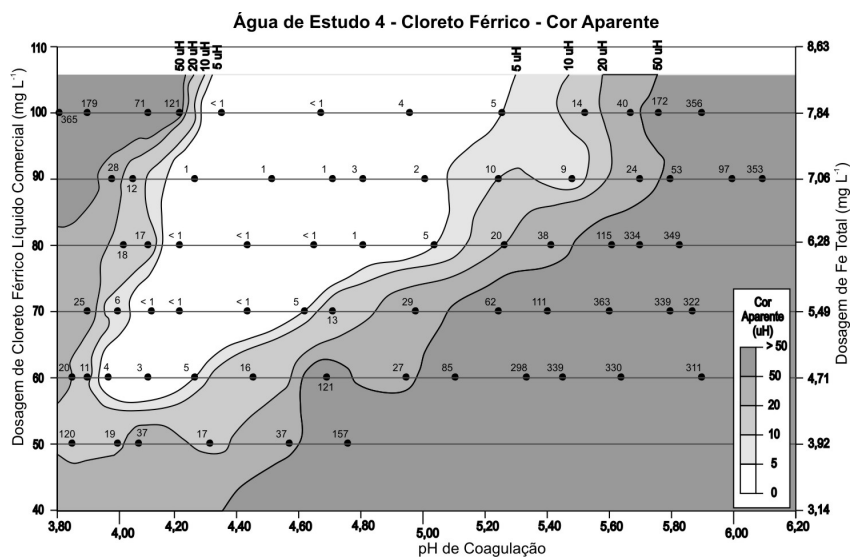

Figura 7. Diagrama de coagulação com cloreto férrico para água de estudo 4 (fração de SHA menor que $30 \mathrm{kDa}$ )

férrico e diferentes valores de $\mathrm{pH}$ de coagulação. Nos diagramas de coagulação, as dosagens de coagulante estão representadas à esquerda em mg L ${ }^{-1}$ do cloreto férrico comercial, à direita em $\mathrm{mg} \mathrm{L}^{-1}$ do ferro total e na abscissa está o pH de coagulação.

Verifica-se pelo diagrama de coagulação na Figura 4 que há duas regiões de coagulação com curvas de cor aparente da água filtrada menor ou igual a 5,0 uH. A menor região foi com $\mathrm{pH}$ de coagulação entre 4,0 e 4,3 e dosagens de cloreto férrico de 30 a $40 \mathrm{mg} \mathrm{L}^{-1}$. A maior região situa-se nos valores de $\mathrm{pH}$ entre 4,8 e 6,0 e dosagens do coagulante de 40 a maiores que $80 \mathrm{mg} \mathrm{L}^{-1}$.

Observa-se nas Figuras 5, 6 e 7 que há apenas uma região de coagulação eficiente (cor aparente da água filtrada $\leq 5 \mathrm{uH}$ ). $\mathrm{Na}$ água de estudo 2 , as dosagens de cloreto férrico comercial foram de 26,4 a maiores que $132 \mathrm{mg} \mathrm{L}^{-1}$ e pH de coagulação entre 3,40 e 6,00. Na água de estudo 3, o pH de coagulação ficou entre 3,70 e 6,00 e as dosagens de coagulante de 30 a maiores que $90 \mathrm{mg} \mathrm{L}^{-1}$ e, na água de estudo 4, o pH ficou entre 3,95 e 5,30 e as dosagens de coagulante de 60 a maiores que $100 \mathrm{mg} \mathrm{L}^{-1}$.

Verificou-se ao empregar as maiores frações de SHA ( $\mathrm{F}_{1}$ : menor que $0,45 \mu \mathrm{m}, \mathrm{F}_{2}$ : entre $100 \mathrm{kDa}$ e $0,45 \mu \mathrm{m} \mathrm{e} \mathrm{F}_{3}$ : entre 30 e $100 \mathrm{kDa}$ ) que a cor aparente da água filtrada resultou menor ou igual a $10 \mathrm{uH}$ a partir de $30 \mathrm{mg} \mathrm{L}^{-1}$ de cloreto férrico comercial; já na fração de menor tamanho molecular aparente $\left(\mathrm{F}_{4}^{\prime}\right.$ : menor que $\left.30 \mathrm{kDa}\right)$, somente apresentou cor aparente igual ou menor a $10 \mathrm{uH}$ a partir da dosagem de $60 \mathrm{mg} \mathrm{L}^{-1}$ de cloreto férrico como produto comercial. Verificou-se neste estudo que quanto menor o tamanho molecular das SHA, maior é a porcentagem de $\mathrm{AF}$ em relação aos $\mathrm{AH}$.

Verifica-se pelos dados da Figura 3 que as frações das SHA de menor tamanho molecular aparente apresentam maior porcentagem de $\mathrm{AF}$ em relação aos AH. Os AF possuem cadeias menores, com estruturas predominantemente alifáticas, maiores teores de grupos funcionais carboxílicos, hidroxilas fenólicas e alcoólicas. Essas características tornam as SHA mais hidrofílicas e mais ácidas. ${ }^{11}$ Isso comprova que não só o tamanho molecular das SHA, mas as características estruturais das frações estudadas influenciaram no processo de coagulação. Portanto, observou-se nos diagramas de coagulação que quanto menor o tamanho molecular, menor foi a remoção de cor aparente da água filtrada.

Comparando-se os resultados obtidos neste trabalho com os obtidos por Dantas et al. ${ }^{17}$ com sulfato de alumínio, conclui-se que o cloreto férrico é mais eficiente para a remoção de cor da água para as frações de SHA estudadas.

\section{CONCLUSÃO}

Com base no presente estudo, concluiu-se que águas com a mesma coloração, mas com substâncias húmicas com propriedades 
estruturais distintas, resultam em diferentes condições de coagulação. Para a água de estudo preparada com a fração de menor tamanho molecular aparente $(<30 \mathrm{kDa})$, as dosagens de coagulante requeridas foram maiores, comprovando que o aumento da fração de AF pode dificultar a coagulação com o cloreto férrico.

Os resultados da caracterização das frações de SHA evidenciam que as frações de menor tamanho molecular aparente são compostas de cadeias menores e estruturas mais alifáticas que aromáticas e maiores teores de grupos oxigenados (grupos funcionais carboxílicos, hidroxilas fenólicas, alcoólicas). Concluiu-se também que as SHA são compostas na maior parte por AF ao contrário das SH extraídas de solo turfoso, que são compostas na maior parte por $\mathrm{AH}$.

\section{AGRADECIMENTOS}

Ao Conselho Nacional de Aperfeiçoamento de Pessoal de Nível Superior (CAPES) e ao Conselho Nacional de Pesquisa e Desenvolvimento $(\mathrm{CNPq})$ pelo apoio financeiro através da concessão de bolsas e auxílios para a realização deste trabalho.

\section{REFERÊNCIAS}

1. Aiken, G. R. Em Humic substances in soil, sediment and water: geochemistry, isolation and characterization; Aiken, G. R.; Mcknight, M. D.; Wershaw, R. L.; MacCarthy, P., eds.; John Wiley: New York, 1985 .

2. Thurman, E. M. Em ref. 1.

3. Edzwald, J. K.; Water Sci. Technol. 1993, 27, 21.

4. Krasner, S. W.; Amy, G.; J. - Am. Water Works Assoc. 1995, 87, 93.

5. Stevenson, F. J. Em ref. 1.

6. Schulten, H. R.; Schnitzer, M.; Naturwissenschaften 1993, 80, 29.

7. Swift, R. S. Em Methods of soil analysis: chemical methods; Sparks, D. L., ed.; SSSA: Madison, 1996.

8. Diallo, M. S.; Simpson, A.; Gassman, P.; Faulon, J. L.; Johnson, J. H.; Goddard, W. A.; Hatcher, P. G.; Environ. Sci. Technol. 2003, 37, 1783.

9. Rocha, J. C.; Rosa, A. H.; Substâncias húmicas aquáticas: interações com espécies metálicas, UNESP: São Paulo, 2003.
10. Thurman, E. M.; Malcolm, R. L.; Environ. Sci. Technol. 1981, 15, 463.

11. Stevenson, F. J.; Humus Chemistry: genesis, composition and reactions, $2^{\text {nd }}$ ed., John Wiley \& Sons: New York, 1994.

12. Di Bernardo L.; Dantas, A. Di B.; Métodos e técnicas de tratamento de água, $2^{\mathrm{a}}$ ed., RiMa: São Carlos, 2005.

13. Thurman, E. M.; Wershaw, R. L.; Malcolm, R. L.; Pinckney, D. J.; Org. Geochem. 1982, 4, 27.

14. Odegaard, H.; Eikebrokk, B.; Storhaug, R.; Water Sci. Technol. 1999, $40,37$.

15. AWWA Coagulation Committee; J. - Am. Water Works Assoc. 1989, 81, 72.

16. Johnson, P. N.; Amirtharajah, A.; J. - Am. Water Works Assoc. 1983, 75, 232.

17. Dantas, A. D. B.; Sloboda, E.; Bernardo, L. Di.; Vieira, E. M.; Resumos do $24^{\circ}$ Congresso de Engenharia Sanitária e Ambiental, Belo Horizonte, Brasil, 2007.

18. Campos, S. X., Azevedo, E. R.; Bonagamba, T. J.; Vieira, E. M.; Di Bernardo, L.; J. Water Supply Res. Technol. Aqua 2007, 56, 327.

19. http://www.ihss.gatech.edu, acessada em Junho 2008.

20. Duarte, M. B. O.; Santos, E. P. H.; Duarte, A. P.; Anal. Chim. Acta 2001, 442, 155.

21. Belzile, N.; Joly, H. A.; Li, H.; Can, J.; Spectroscopy 1997, 75, 14.

22. Huffman, E. W. D. JR.; Stuber, H. A. Em ref. 1.

23. Campanella, L.; Petronio, B. M.; Braguglia, C.; Int. J. Anal. Chem. 1994, 60,60 .

24. Rosa, A. H.; Rocha, J. C.; Furian, M.; Quim. Nova 2000, 23, 476.

25. Ricca, G.; Severini, F.; Di Silvestro, G.; Yuan, C. M.; Adani, F.; Geoderma 2000, 98, 115.

26. Peuravuori, J.; Pihlaja, K.; Anal. Chim. Acta 1997, 337, 133.

27. Cook, R. L.; Anal. Bioanal. Chem. 2004, 378, 1484.

28. Piccolo, A.; Soil Sci. 2001, 166, 810.

29. Piccolo, A.; Stevenson, F. J.; Geoderma 1982, 27, 195.

30. MacCarthy, P.; Rice, J. A. Em ref. 1.

31. Lu, X. Q.; Hanna, J. V.; Johnson, W. D.; Appl. Geochem. 2000, 15, 1019.

32. Malcom, L. C., Anal. Chim. Acta 1990, 232, 19. 